\title{
Cell-penetrating Peptide-coated Liposomes for Drug Delivery Across the Blood-Brain Barrier
}

\author{
BO YUAN ${ }^{1}$, YARONG ZHAO ${ }^{2}$, SHIYAN DONG ${ }^{2}$, YATING SUN ${ }^{2}$, FEI HAO ${ }^{2}$, JING XIE ${ }^{2}$, \\ LESHENG TENG ${ }^{2}$, ROBERT J. LEE ${ }^{2,3}$, YAOWEN FU ${ }^{1}$ and YE BI ${ }^{2,4}$ \\ ${ }^{1}$ Department of Urology, The First Hospital of Jilin University, Changchun, P.R. China; \\ ${ }^{2}$ School of Life Sciences, Jilin University, Changchun, P.R. China; \\ ${ }^{3}$ College of Pharmacy, Division of Pharmaceutics, The Ohio State University, Columbus, OH, U.S.A.; \\ ${ }^{4}$ Practice Training Center, Changchun University of Chinese Medicine, Changchun, P.R. China
}

\begin{abstract}
Background/Aim: Glioma is a deadly form of brain cancer. Doxorubicin is cytotoxic against glioma cells. However, the blood-brain barrier (BBB) limits its ability to be delivered to the brain. Materials and Methods: Liposomes (R8PLP) formed from, 1,2-dioleoyl-3-trimethylammonium-propane chloride (DOTAP), 1,2-distearoyl-sn-glycero-3-phosphoethanolamine- $N$ [methoxy-(polyethylene glycol)-2000] (PEG-DSPE), cholesterol and egg phosphatidylcholine (ePC) were modified by cellpenetrating peptide $R 8$ conjugated with oleic acid as a novel method for delivering doxorubicin. The antitumor effect of R8PLP was evaluated by uptake, cytotoxicity and brain accumulation. Results: The size of R8PLP was $95 \mathrm{~nm}$. Doxorubicin was loaded into R8PLP by active loading with more than $95 \%$ encapsulation efficiency. Cellular uptake of R8PLP by U87-MG cells was 8.6-fold higher than that of unmodified liposomes. R8PLP reduced cell viability by $16.18 \%$ and $18.11 \%$ compared to cholesterol-ePC-liposomes and free doxorubicin, respectively, at $3.6 \mu \mathrm{M}$ after $24 \mathrm{~h}$ treatment. The biodistribution of doxorubicin in the brain was significantly improved by R8PLP. The area under the concentration-time curve $\left(A U C_{0.5-12} h\right)$ of R8PLP was 2.4-times higher than that of cholesterol-ePC-PEGDSPE-liposomes. Conclusion: These results suggest that R8conjugated oleic acid-modified liposomes are effective delivery vehicles for glioma.
\end{abstract}

Correspondence to: Robert J. Lee, College of Pharmacy, The Ohio State University, Columbus, Ohio 43210, U.S.A. Tel: +1 6142924172, Fax: +8643185155320, e-mail: lee.1339@osu.edu or Yaowen Fu, Department of Urology, The First Hospital of Jilin University, 71 Xinmin Ave, Changchun, Jilin, China. Tel: +86 43181875831, Fax: +8643181875831, e-mail: fuyaowen@medmail.com.cn or Ye Bi, Practice Training Center, Changchun University of Chinese Medicine, 1035 Boshuo Rd, Changchun, Jilin, China. Tel: +86 43186763979, Fax: +86 43186763979, e-mail: biye88@outlook.com

Key Words: Glioma, BBB, doxorubicin, drug delivery, cellpenetrating peptide, liposomes.
Glioma is an aggressive malignant tumor in the brain, with high morbidity and mortality (1-3). The survival time of patients with glioma after diagnosis is about 12-15 months (4). Treatment options for glioma are limited; surgery is associated with recurrence $(5,6)$. Chemotherapy using agents for glioma is limited to temozolomide, which has limited efficacy. Doxorubicin is a potential treatment option (7). Unfortunately, the blood-brain barrier (BBB) limits drug delivery to the brain $(8,9)$, diminishing its effectiveness.

Liposomes are nontoxic and nonimmunogenic drug delivery vehicles that have been used for doxorubicin delivery. However, delivery of liposomes across the BBB remains a great challenge.

Cell-penetrating peptides are a class of short peptides shown to facilitate cellular uptake of biomolecules (10). In this study, liposomes were modified with a cell-penetrating peptide, octa-arginine (R8), which has been shown to improve brain delivery of agents in vivo $(11,12)$. Oleic acid was used to modify R8 to facilitate liposomal incorporation.

\section{Materials and Methods}

Materials. R8 was synthesized by GL Biochem Ltd. (Shanghai, China). 1,2-Dioleoyl-3-trimethylammonium-propane chloride (DOTAP) and 1,2-distearoyl-sn-glycero-3-phosphoethanolamine- $N$ [methoxy-(polyethylene glycol)-2000] (PEG-DSPE) were purchased from Lipoid (Newark, NJ, USA). Egg phosphatidylcholine (ePC) was obtained from Avanti Polar Lipids Inc. (Alabaster, AL, USA). Oleic acid was obtained from Sigma (St. Louis, MO, USA). Modified Eagle's medium (MEM) and fetal bovine serum (FBS) were purchased from HyClone (Logan, UT, USA). 4',6-Diamidino-2-phenylindole (DAPI) was obtained from Molecular Probes Inc. (Eugene, OR, USA). 3-(4,5-Dimethylthiazol-2-yl)-2,5-diphenyltetrazolium bromide (MTT) was purchased from Sigma-Aldrich (St. Louis, MO, USA). U87-MG, a glioblastoma cell line, was obtained from the American Type Culture Collection (Manassas, VA, USA).

Preparation and characterization of liposomes. R8-conjugated oleic acid (R8-OA) was synthesized according to a previously reported 
Table I. Composition and characteristics of liposomal formulations $(n=3)$.

\begin{tabular}{lcccc}
\hline Formulation & \multicolumn{1}{c}{ Composition } & Ratio (molar) & Diameter (nm) & $\zeta$-Potential (mV) \\
\hline R8LP & DOTAP, R8-OA, ePC, cholesterol & $20 / 25 / 18 / 35$ & $91.1 \pm 2.3$ & $+20.7 \pm 5.6$ \\
R8PLP & DOTAP, R8-OA, ePC, cholesterol, PEG-DSPE & $20 / 25 / 18 / 32 / 3$ & $95.4 \pm 5.1$ & $+12.1 \pm 4.0$ \\
PLP & ePC, cholesterol, PEG-DSPE & $65 / 30 / 5$ & $92.5 \pm 3.8$ & $-7.7 \pm 2.9$ \\
LP & ePC, cholesterol & $65 / 35$ & $81.3 \pm 4.2$ & $-10.4 \pm 3.3$ \\
\hline
\end{tabular}

DOTAP: 1,2-Dioleoyl-3-trimethylammonium-propane chloride; R8-OA: R8-conjugated oleic acid; ePC: egg phosphatidylcholine; PEG-DSPE: 1,2distearoyl-sn-glycero-3-phosphoethanolamine- $N$-[methoxy-(polyethylene glycol)-2000].

method (13). A series of liposomes with were prepared according to Table I (14): LP: Liposomes of ePC-cholesterol; PLP: liposomes of PEG-DSPE-LP; R8LP: R8-conjugated oleic acid liposomes of DOTAP-LP; R8PLP: R8-conjugated oleic acid liposomes of PLP. The composition of liposomes was adjusted to optimize uptake by U87-MG cells. Liposomes were prepared by an ethanol injection method and doxorubicin was actively loaded into liposomes by an ammonium sulfate gradient. Firstly, R8-OA, ePC, cholesterol, and PEG-DSPE were dissolved in ethanol at different ratios to form a mixture. This was then injected into ammonium sulfate $(250 \mathrm{mM})$ at a volume ratio of 1:10 to form liposomes. Liposomes were dialyzed to remove the ammonium sulfate external to the liposomes. Finally, doxorubicin was added to initiate drug loading at final concentration of $1 \mathrm{mg} / \mathrm{ml}$. The mean size and $\zeta$-potential of liposomes were measured by Zetasizer Nano ZS 90 from Malvern Instruments, Ltd. (Malvern, U.K.). Surface morphology of liposomes was examined on a JEOL scanning electron microscope (SEM) (Tokyo, Japan). The encapsulation efficiency (EE\%) of doxorubicin in liposomes was then evaluated.

The release of doxorubicin in vitro. A dialysis method was used to study the release of doxorubicin from liposomes. Briefly, liposomes were placed into dialysis tubes (molecular weight cut-off of 8,000 $12,000 \mathrm{Da})$. The external solution for dialysis was phosphatebuffered saline (PBS). The dialysis was performed in the dark with stirring at $37^{\circ} \mathrm{C}$. The external solution was periodically sampled and replaced with an equal volume of PBS.

The stability of R8PLP was evaluated in PBS with $10 \%$ FBS at $37^{\circ} \mathrm{C}$. Liposomes were incubated for $0,2,4,8,12$ or $24 \mathrm{~h}$ and measured the size and EE\% of liposomes (15). The encapsulated doxorubicin $\left(\mathrm{E}_{\mathrm{Dox}}\right)$ in R8PLP was separated from liposomes by Sephadex G-50 (Yuanye Biological Technology Co.,Ltd., Shanghai, China). The total doxorubicin $\left(\mathrm{T}_{\text {Dox }}\right)$ was released from liposomes by Triton X-100 (Yuanye Biological Technology Co.,Ltd., Shanghai, China). The EE\% was calculated using the following equation: $\mathrm{EE} \%=\mathrm{E}_{\text {Dox }} / \mathrm{T}_{\text {Dox }} \times 100 \%$

The concentration of doxorubicin was quantified by Agilent 1100 high-performance liquid chromatography (Santa Clara, CA, USA) using a fluorescence detector at Ex/Em of 480/525 nm using acetonitrile/water (50:50) as the mobile phase.

Cellular uptake of liposomes in vitro. Laser confocal microscopy was used to qualitatively analyze U87-MG cellular uptake. U87-MG cells were seeded into glass-bottom culture dish at a density of $1 \times 10^{5}$ cells/well and cultured for $24 \mathrm{~h}$ at $37^{\circ} \mathrm{C}$ in $5 \% \mathrm{CO}_{2}$ atmosphere. Liposomes were then added to the U87-MG cells, which were then incubated for $4 \mathrm{~h}$. The cells were fixed in $4 \%$ paraformaldehyde for 10 mins, washed with PBS. The nucleus was stained with $2 \mu \mathrm{g} / \mathrm{ml}$ of DAPI for $10 \mathrm{~min}$. Finally, cells were washed with PBS three times and observed under a confocal microscopy from Carl Zeiss (Oberkochen, Jena, Germany) on the red channel.

The cellular uptake of liposomes was further quantitatively measured by flow cytometry. U87-MG cells were plated into 6-well plates at the density of $1 \times 10^{5}$ cells/well and cultured for $24 \mathrm{~h}$ at $37^{\circ} \mathrm{C}$ in $5 \% \mathrm{CO}_{2}$ atmosphere. Liposomes were then added to the U87-MG cells, which were incubated for $4 \mathrm{~h}$. The cells were fixed with $4 \%$ paraformaldehyde for 10 mins and washed with PBS. Fluorescence intensity of cells was measured on a flow cytometer from Beckman Coulter (Brea, CA, USA).

Cytotoxicity assay. Briefly, $1 \times 10^{4}$ cells per well were seeded into 96-well plates at $37^{\circ} \mathrm{C}$ and cultured for $24 \mathrm{~h}$. Liposomes with doxorubicin concentration of $3.6 \mu \mathrm{M}$ were added and the cells were incubated for 24, 48 and $72 \mathrm{~h}$ after treatment. Ten microliters of MTT $(5 \mathrm{mg} / \mathrm{ml})$ was added and the plates were incubated for $4 \mathrm{~h}$. Formazan was dissolved in $150 \mu \mathrm{l}$ dimethylsulfoxide and the absorbance at $490 \mathrm{~nm}$ measured by on a BioTek Synergy4 microplate reader (Winooski, VT, USA).

Animals. In order to determine whether R8-OA-modified liposomes were able to penetrate the BBB and increase doxorubicin accumulation in the brain, an in vivo study was carried out in mice $(16,17)$. Female KM mice $(30 \pm 2 \mathrm{~g})$ were purchased from Beijing Vital River Laboratory Animal Technology Co., Ltd. (Beijing, China). All animal experiments were approved by the Animal Ethics Committee of the Jilin University (No. 201805010). Mice were separated into two groups of 21 each. Mice were fasted for $12 \mathrm{~h}$ before administering liposomes. Mice received a single dose of liposomes $(3 \mathrm{mg} / \mathrm{kg}$ of doxorubicin) through tail vein injection. Mice were sacrificed and the brain was collected at $0.5,1,2,4,6,8$, or $12 \mathrm{~h}$. Tissue samples were used for analysis of doxorubicin content.

Distribution of doxorubicin in the brain. Doxorubicin analysis in brain tissue was established by Waters Ultra-high performance liquid chromatography-tandem mass spectrometry (UPLC-MS/MS) (Milford, MA, USA) using daunorubicin as internal standard (IS). The method was reported previously (18). Briefly, brain tissue was homogenized with two volumes of water, IS was added to $100 \mu \mathrm{l}$ homogenization solution at a final concentration of $20 \mathrm{ng} / \mathrm{ml}$. Samples were treated with $200 \mu$ l borate buffer (6\% of w/v, $\mathrm{pH} 9.5$ ) for 2 mins under vortex. Doxorubicin and IS were extracted from the mixture by $1 \mathrm{mI}$ of chloroform/1-propanol $(4: 1, \mathrm{v} / \mathrm{v})$ for $2 \mathrm{~min}$ 


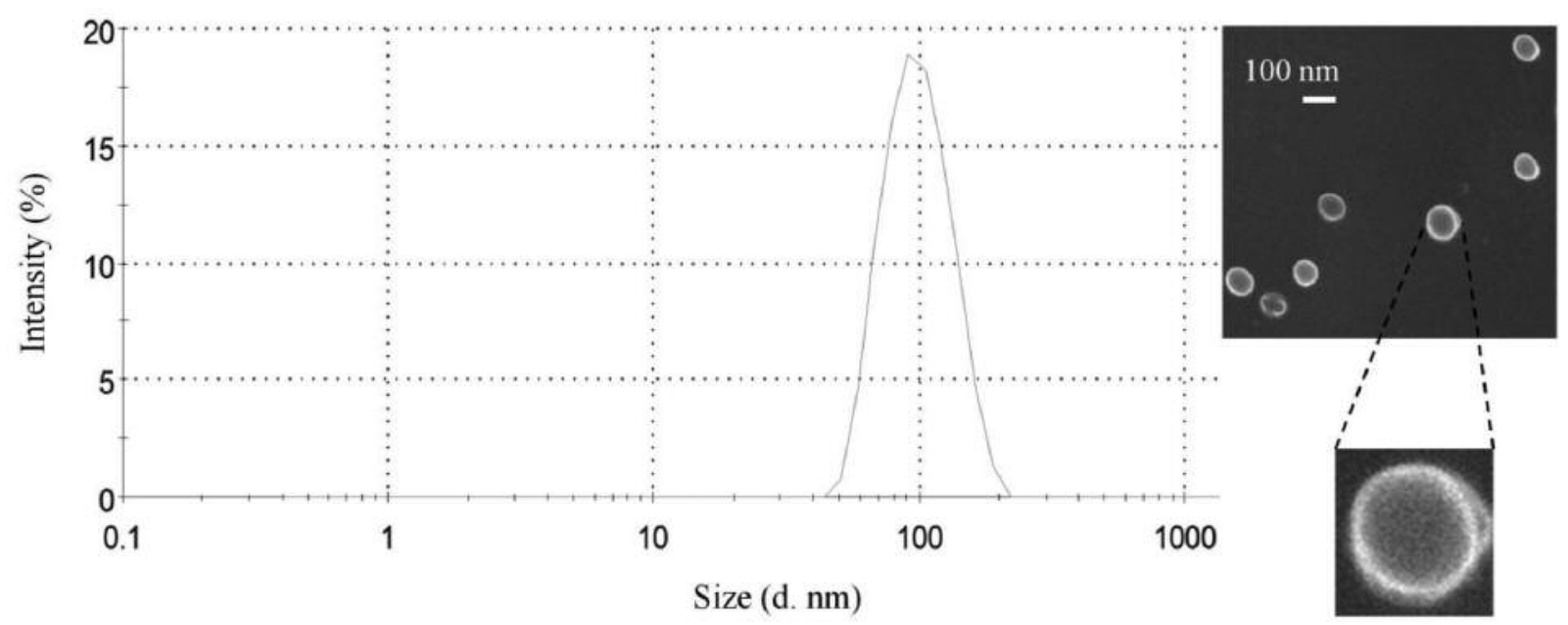

Figure 1. Characterization of R8-conjugated oleic acid liposomes of 1,2-dioleoyl-3-trimethylammonium-propane chloride-egg phosphatidylcholinecholesterol-1,2-distearoyl-sn-glycero-3-phosphoethanolamine-N-[methoxy-(polyethylene glycol)-2000] (R8PLP). The diameter and morphology of R8PLP were characterized. The size of R8PLP was measured in intensity-weighted Gaussian size distribution mode, the polydispersity index was 0.133. Scanning electron microscopy image was obtained in secondary electron imaging mode: $3.0 \mathrm{kV}, \times 30,000$.

with mixing and the organic phase was separated and dried by nitrogen. The residue was resuspended by the initial mobile phase and $10 \mu \mathrm{l}$ of sample was injected into the UPLC-MS/MS system.

UPLC-MS/MS conditions: Source temperature $150^{\circ} \mathrm{C}$, capillary voltage $3 \mathrm{kV}$, cone voltage $30 \mathrm{~V}$, and desolvation temperature was set at $500^{\circ} \mathrm{C}$. Doxorubicin was measured by quantitative ions of $\mathrm{m} / \mathrm{z}$ $544.20 \rightarrow 397.21$ and $\mathrm{m} / z \quad 528.18 \rightarrow 321.19$ for doxorubicin and IS, respectively.

Statistical analysis. The data are described as the mean \pm SD. The statistical significance of results was calculated by $t$-test. A $p$-value of less than 0.05 was accepted as indicating a significant difference.

\section{Results and Discussion}

Preparation and characterization of liposomes. The size distribution and $\zeta$-potential of liposomes are shown in Table I. Mean size of liposomes was from $81.3 \mathrm{~nm}$ to $95.4 \mathrm{~nm}$. The size of liposomes composed of ePC-cholesterol (LP) was 81.3 $\mathrm{nm}$, liposomes were larger when they contained PEG-DSPE, DOTAP or R8-OA. R8LP had a larger pdipolydispersity index (0.32) than other liposomes. The size of R8PLP was $95.4 \mathrm{~nm}$ with pdipolydispersity index of 0.16 and the $\zeta$-potential of R8PLP was $+12.1 \mathrm{mV}$. LP and liposomes of ePC-cholesterolPEG-DSPE (PLP) exhibited negative surface charge. However, the $\zeta$-potentials of R8LP and R8PLP were positive. SEM was used to further characterize the surface morphology of liposomes. An SEM image of R8PLP is shown in Figure 1. R8PLP had typical liposome features and exhibited a spherical structure with a smooth surface.

Doxorubicin was actively loaded into liposomes using an ammonium sulfate gradient as driving force. The liposomes

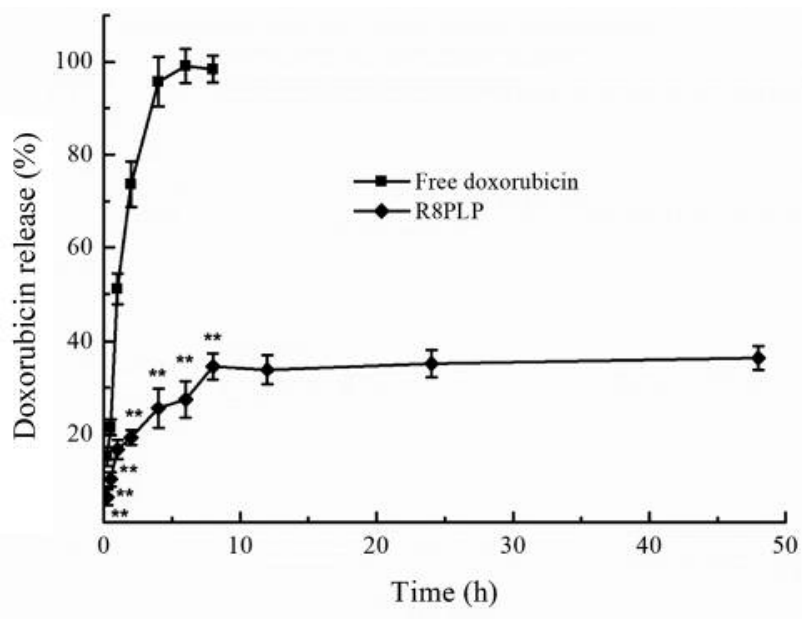

Figure 2. Release of doxorubicin in phosphate-buffered saline. Cumulative release of free doxorubicin and doxorubicin from R8-conjugated oleic acid liposomes of 1,2-dioleoyl-3-trimethylammonium-propane chloride-egg phosphatidylcholine-cholesterol-1,2-distearoyl-sn-glycero-3phosphoethanolamine-N-[methoxy-(polyethylene glycol)-2000] (R8PLP) were measured by high-performance liquid chromatography after incubating with phosphate-buffered saline ( $\mathrm{pH} 7.4)$ in a dialysis tube. **Significantly different at $p<0.01$ (versus free doxorubicin).

had a high EE\% for doxorubicin. EE\% of liposomes was more than $92 \%$. The EE\% of R8PLP was $95.3 \%$.

Release and stability of doxorubicin in vitro. As shown in Figure 2, only $25.6 \%$ of doxorubicin in R8PLP was released at $4 \mathrm{~h}$ and $33.9 \%$ at $12 \mathrm{~h}$. As shown in Figure 3, the size of R8PLP increased to $111.1 \mathrm{~nm}$ and the EE\% decreased by 

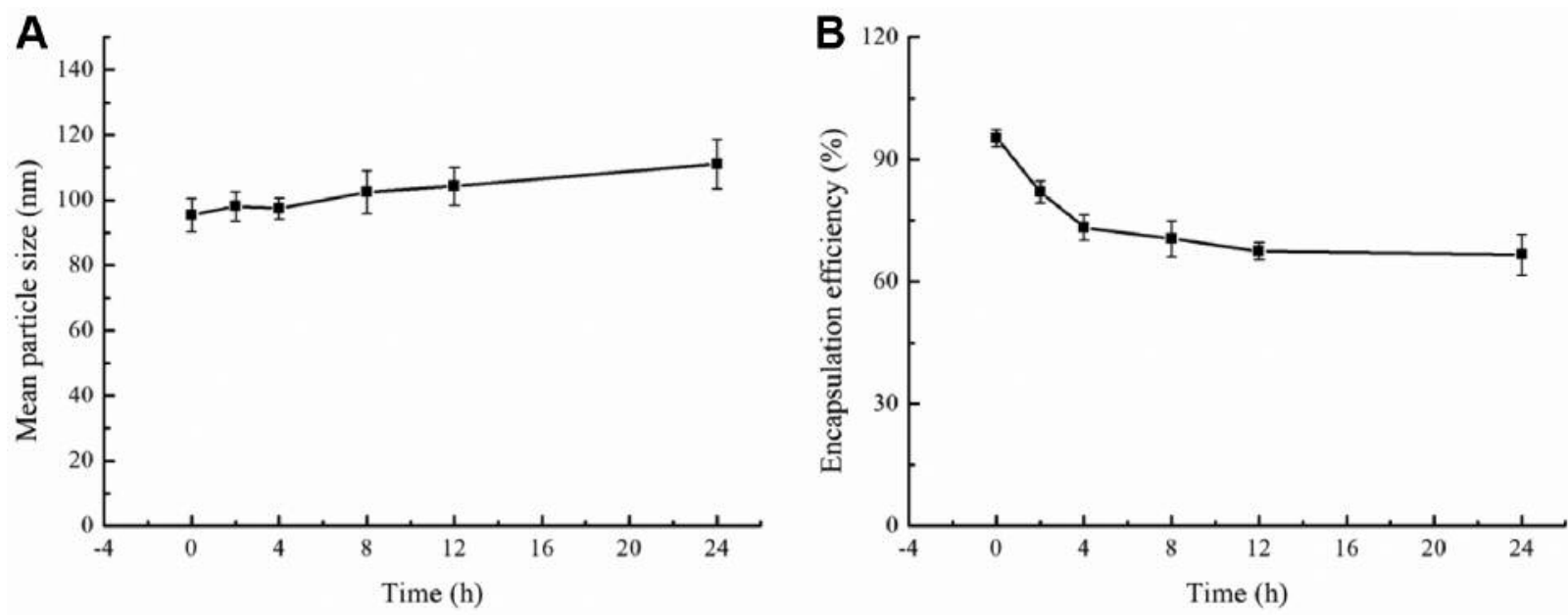

Figure 3. The colloidal stability of R8-conjugated oleic acid liposomes of 1,2-dioleoyl-3-trimethylammonium-propane chloride-egg phosphatidylcholinecholesterol-1,2-distearoyl-sn-glycero-3-phosphoethanolamine-N-[methoxy-(polyethylene glycol)-2000] (R8PLP) in fetal bovine serum (FBS). The stability of R8PLP in FBS was evaluated, by particle size (A) and encapsulation efficiency (EE\%) (B). There was no significant change in the size of R8PLP after 24 incubation with FBS, but EE\% was significantly different at $24 \mathrm{~h}$ compared to $0 \mathrm{~h}$. R8PLP was stable in serum.

almost $28.7 \%$ within $24 \mathrm{~h}$ at $37^{\circ} \mathrm{C}$ in $10 \% \mathrm{FBS}$. R8PLP was relatively stable in the presence of serum.

Cellular uptake of liposomes in vitro. Laser confocal microscopy was used to qualitative analyze the cellular uptake of liposomes. Nuclei and doxorubicin were observed in DAPI and doxorubicin channels, respectively. As shown in Figure 4, red spots in the doxorubicin channel indicated that U87-MG cells had internalized liposomes. LP- and PLP-treated cells exhibited relatively lower fluorescence intensity than cells treated with R8LP and R8PLP. These results showed that R8-OA promoted the cellular uptake of liposomes.

The uptake of liposomes by U87-MG cells were also quantitative analyzed by flow cytometry, as shown in Figure 5. The uptake fluorescence intensity was consistent with the confocal microscopy data. The cellular uptake of PLP was similar to that of LP, which was significantly lower than those of R8LP and R8PLP. The fluorescence intensities of R8PLP- and R8LP-treated cells were greater than those treated with LP, which indicated that R8-OA facilitated liposomal uptake by U87-MG cells.

Cytotoxicity assay. Cytotoxicity was investigated by the MTT assay. LP, R8LP, and R8PLP all exhibited similar or superior cytotoxicity than free doxorubicin. R8-OAmodified liposomes significantly improved doxorubicin cytotoxicity against U87-MG cells compared to PLP and LP. As can be seen in Figure 6, cell viability after R8LP or R8PLP treatment decreased relative to that of cells treated with free doxorubicin at $3.6 \mu \mathrm{M}$ at $24 \mathrm{~h}$. The cell-killing effect of liposomes was greater at 48 and $72 \mathrm{~h}$. These results suggest that U87-MG cells were sensitive to doxorubicin and R8-OA-modified liposomes improved the cytotoxicity of doxorubicin.

Distribution of doxorubicin in the brain. Doxorubicin delivery to the brain using R8PLP was quantified. The concentration of doxorubicin in the homogenized brain was defined as $X$, peak area ratio of doxorubicin and IS was defined as $Y$, and $1 / X^{2}$ was used as the weighting factor. The method had a good linear relation at concentrations from 5 to $1,000 \mathrm{ng}(Y=0.273102 X+0.003257, \mathrm{R}>0.99)$. Brain distribution of doxorubicin delivered by R8PLP and PLP are shown in Figure 7. R8PLP significantly accumulated in the brain compared to PLP, the area under the concentrationtime curve $\left(\mathrm{AUC}_{0.5-12 \mathrm{~h}}\right)$ of R8PLP was 2.4 times that of PLP. However, the half-life $\left(\mathrm{t}_{1 / 2}\right)$ of R8PLP was similar to that of PLP (3.47 versus $3.94 \mathrm{~h}$ ). These results showed that R8-OA-modified liposomes exhibited superior BBB penetration and brain accumulation.

\section{Discussion}

The results indicated that R8-OA was able to be incorporated into liposomes loaded with doxorubicin without adversely affecting their stability. The resulting R8PLP was efficiently taken up by tumor cells and was able to deliver doxorubicin to the brain. This might be due to the positive charge on the modified liposomes, which can facilitate internalization of the liposomes by tumor cells and transcytosis of the liposomes across the BBB. Increased brain distribution of 


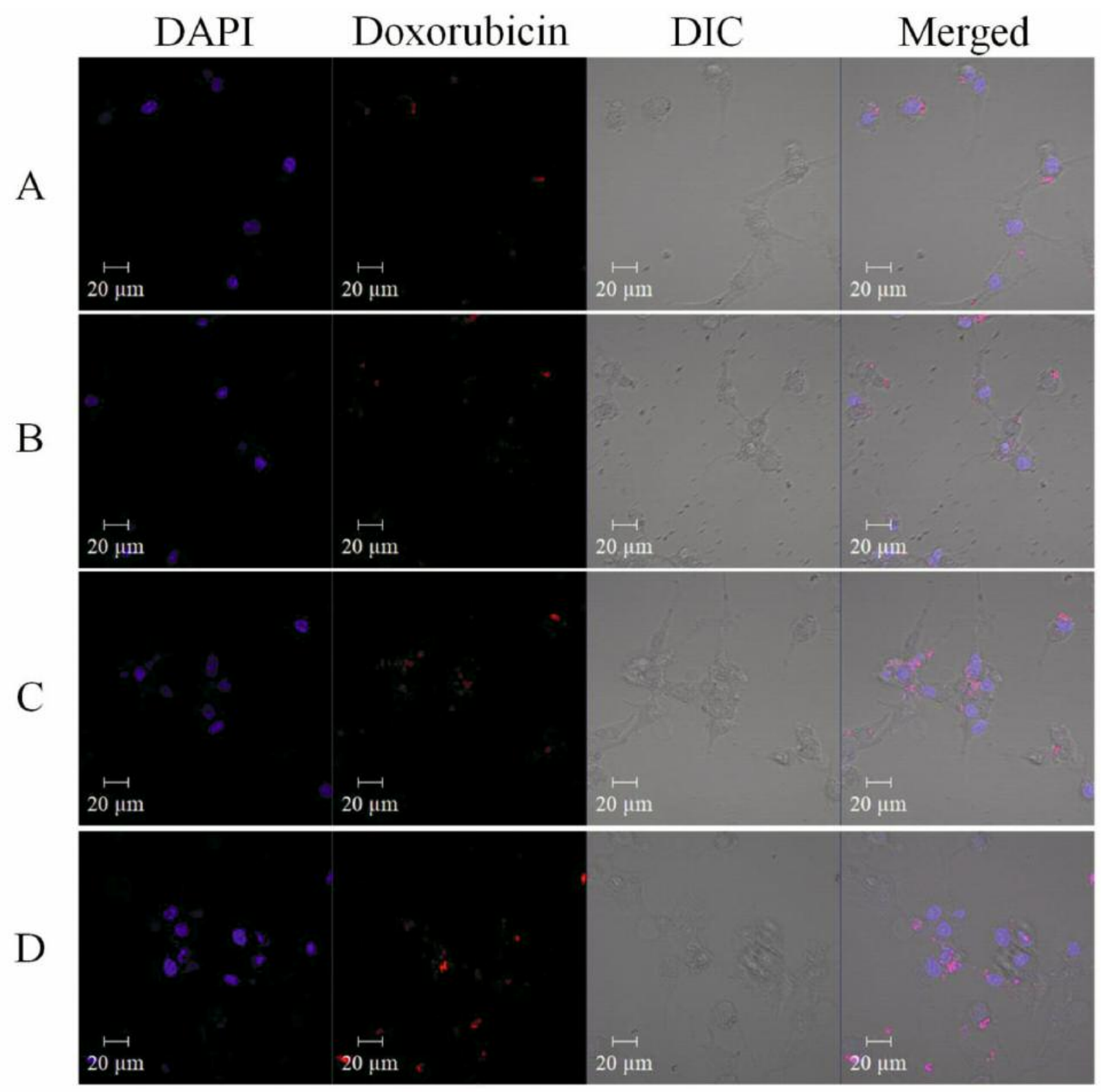

Figure 4. Uptake of liposomes by U87-MG cells. Fluorescence uptake intensity of liposomes was observed by confocal microscopy. A: LP: Egg phosphatidylcholine (ePC)-cholesterol; B: PLP: ePC-cholesterol-1,2-distearoyl-sn-glycero-3-phosphoethanolamine- $N$-[methoxy-(polyethylene glycol)-2000] (PEG-DSPE); C: R8LP: R8-conjugated oleic acid-1,2-dioleoyl-3-trimethylammonium-propane chloride (D0TAP)-LP; D: R8PLP: R8-conjugated oleic acid liposomes of PLP. Nuclei were stained by 4',6-diamidino-2-phenylindole (DAPI) to exhibit blue fluorescence, doxorubicin emitted red fluorescence. DIC: Differential interference contrast mode to display cellular morphology.

doxorubicin suggests that these liposomes may have the potential for achieving enhanced therapeutic efficacy against intracranial tumors.

In future studies, we plan to investigate the effect of the $\mathrm{R} 8$ modification on the plasma pharmacokinetics and cardiotoxicity of liposomal doxorubicin, as well as therapeutic efficacy against intracranial glioma xenografts.

\section{Conclusion}

R8-OA modified liposomes were developed to improve the BBB-penetrating ability of doxorubicin. Cellular uptake, cytotoxicity and brain distribution of doxorubicin were enhanced with R8PLP compared to LP. R8PLP is a promising drug carrier and has potential applications in glioma therapy. 


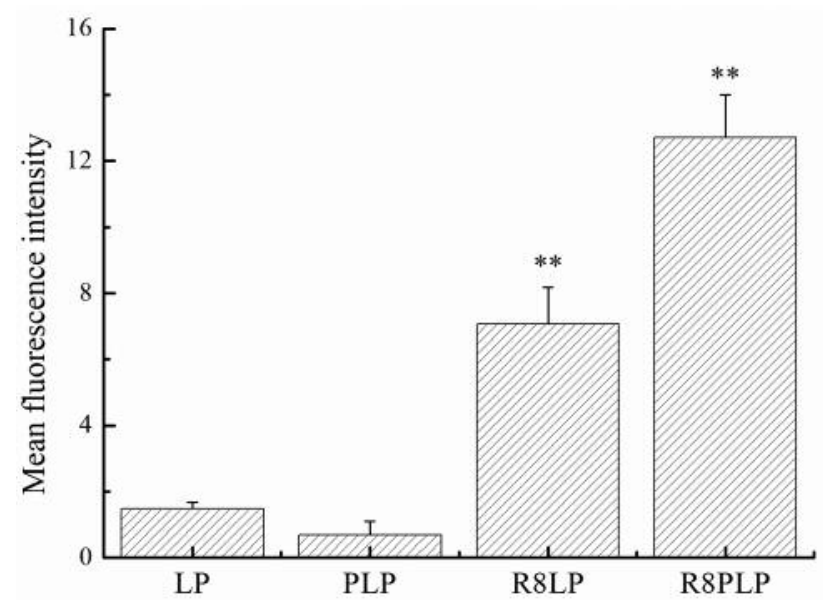

Figure 5. Cellular uptake of liposomes measured by flow cytometry. R8conjugated oleic acid significantly improved cellular uptake of liposomes compared to traditional liposomes. LP: Egg phosphatidylcholine (ePC)cholesterol; PLP: ePC-cholesterol-1,2-distearoyl-sn-glycero-3-phosphoethanolamine-N-[methoxy-(polyethylene glycol)-2000] (PEG-DSPE); R8LP: R8-conjugated oleic acid-1,2-dioleoyl-3-trimethyla-mmoniumpropane chloride (DOTAP)-LP; R8PLP: R8-conjugated oleic acid liposomes of PLP. **Significantly different at $p<0.01$ versus $L P$.

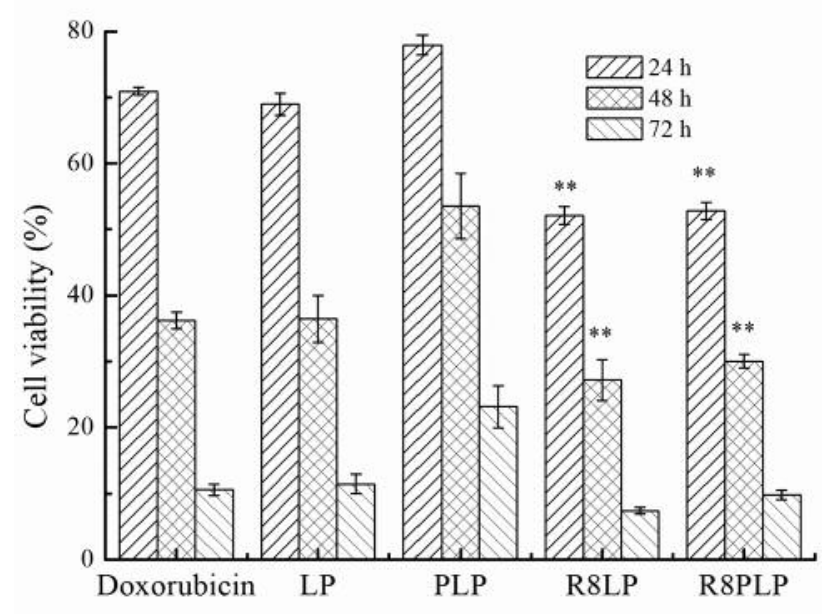

Figure 6. Cytotoxicity of liposomes. MTT assay was used to determine cytotoxicity in U87-MG cells for 24, 48 and 72 h. LP: Egg phosphatidylcholine (ePC)-cholesterol; PLP: ePC-cholesterol-1,2distearoyl-sn-glycero-3-phosphoethanolamine- $N$-[methoxy-(polyethylene glycol)-2000] (PEG-DSPE); R8LP: R8-conjugated oleic acid-1,2dioleoyl-3-trimethylammonium-propane chloride (DOTAP)-LP; R8PLP: $R 8$-conjugated oleic acid liposomes of PLP. **Significantly different at $p<0.01$ versus free doxorubicin.

\section{References}

1 Ruan S, Xiao W, Hu C, Zhang H, Rao J, Wang S, Wang X, He $\mathrm{Q}$ and Gao H: Ligand-mediated and enzyme-directed precise targeting and retention for enhanced treatment of glioblastoma. ACS Appl Mater Interfaces 9(24): 20348-20360, 2017.

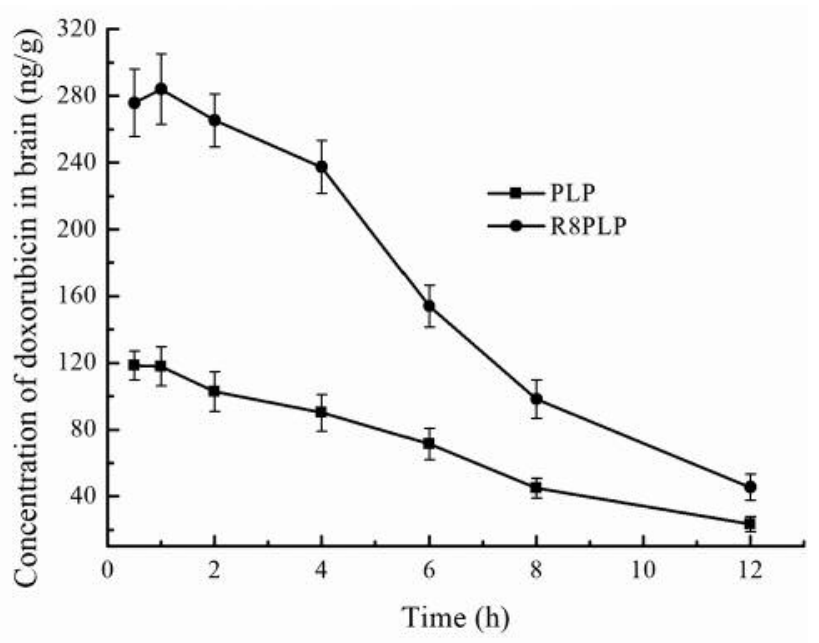

Figure 7. Distribution of doxorubicin in the brain of mice. The concentration of doxorubicin in the brain was analyzed by ultra-high performance liquid chromatography-tandem mass spectrometry $(n=3)$. PLP: Liposomes of egg phosphatidylcholine-cholesterol-1,2-distearoylsn-glycero-3-phosphoethanolamine- $N$-[methoxy-(polyethylene glycol)2000]; R8PLP: R8-conjugated oleic acid liposomes of 1,2-dioleoyl-3trimethylammonium-propane chloride-PLP.

2 Cohen ZR, Ramishetti S, Peshesyaloz N, Goldsmith M, Wohl A, Zibly Z and Peer D: Localized RNAi therapeutics of chemoresistant grade IV glioma using hyaluronan-grafted lipid-based nanoparticles. ACS Nano 9(2): 1581, 2015.

3 Séhédic, Delphine, Cikankowitz, Annabelle, Hindré, François, François, Garcion and Emmanuel: Nanomedicine to overcome radioresistance in glioblastoma stem-like cells and surviving clones. Trends Pharmacol Sci 36(4): 236-252, 2015.

4 Balça-Silva J, Carmo AD, Tão H, Rebelo O, Barbosa M, MouraNeto V, Sarmento-Ribeiro AB, Lopes MC and Moreira JN: Nucleolin is expressed in patient-derived samples and glioblastoma cells, enabling improved intracellular drug delivery and cytotoxicity. Exp Cell Res 370(1): 68-77, 2018.

5 Ong BY, Ranganath SH, Lee LY, Lu F, Lee HS, Sahinidis NV and Wang $\mathrm{CH}$ : Paclitaxel delivery from PLGA foams for controlled release in post-surgical chemotherapy against glioblastoma multiforme. Biomaterials 30(18): 3189-3196, 2009.

6 Chen C, Duan Z, Yan Y, Li R, Liang P, Liang J, Xu X and Wang $\mathrm{J}$ : Peptide-22 and cyclic RGD functionalized liposomes for glioma targeting drug delivery overcoming BBB and BBB. ACS Appl Mater Interfaces 9(7): 5864, 2017.

7 Yu C, Morshed R, Auffinger B, Tobias AL and Lesniak MS: Multifunctional nanoparticles for brain tumor diagnosis and therapy. Adv Drug Deliv Rev 66(1): 42-57, 2014.

8 Sarin H: Recent progress towards development of effective systemic chemotherapy for the treatment of malignant brain tumors. J Transl Med 7(1): 77, 2009.

9 Kamran N, Calinescu A, Candolfi M, Chandran M, Mineharu Y, Assad AS, Koschmann C, Nunez F, Lowenstein P and Castro M: Recent advances and future of immunotherapy for glioblastoma. Expert Opin Biol Ther 16(10): 1245, 2016. 
10 Lai F, Fadda AM and Sinico C: Liposomes for brain delivery. Expert Opin Drug Deliv 10(7): 1003-1022, 2013.

11 Liu Y, Ran R, Chen J, Kuang Q, Tang J, Mei L, Zhang Q, Gao $\mathrm{H}$, Zhang $\mathrm{Z}$ and He Q: Paclitaxel-loaded liposomes decorated with a multifunctional tandem peptide for glioma targeting. Biomaterials 35(17): 4835-4847, 2014.

12 Kamei N, Yamaoka A, Fukuyama Y, Itokazu R and Takedamorishita M: Noncovalent strategy with cell-penetrating peptides to facilitate the brain delivery of insulin through the blood-brain barrier. Mol Cell Neurosci 41(4): 546-554, 2018.

13 Yuhuan L, Yujing L, Xinmei W, Lee RJ and Lesheng T: Fatty acid modified octa-arginine for delivery of siRNA. Int $\mathrm{J}$ Pharmaceut 495(1): 527-535, 2015.

14 Wang X, Yu B, Ren W, Mo X, Zhou C, He H, Jia H, Wang L, Jacob ST and Lee RJ: Enhanced hepatic delivery of siRNA and microRNA using oleic acid based lipid nanoparticle formulations. J Contr Release 172(3): 690-698, 2013.

15 Zong T, Mei L, Gao H, Shi K, Chen J, Wang Y, Zhang Q, Yang $\mathrm{Y}$ and $\mathrm{He} \mathrm{Q}$ : Enhanced glioma targeting and penetration by dualtargeting liposome co-modified with T7 and TAT. JPharmaceut Sci 103(12): 3891, 2014
16 Labussiere M, Aarnink A, Pinel S, L, Escanye J, Barberi-Heyob $\mathrm{M}$, Bernier-Chastagner V, Plenat F and Chastagner P: Interest of liposomal doxorubicin as a radiosensitizer in malignant glioma xenografts. Anticancer Drugs 19(10): 991, 2008.

17 Zheng C, Ma C, Bai E, Yang K and Xu R: Transferrin and cellpenetrating peptide dual-functioned liposome for targeted drug delivery to glioma. Int J Clin Exp Med 8(2): 1658, 2015.

18 Liu Z, Ye B, Sun Y, Fei H, Lu J, Meng Q, Lee RJ, Tian Y and Jing X: Pharmacokinetics of a liposomal formulation of doxorubicin in rats. Saudi Pharmaceut J 25(4): 531-536, 2017.
Received October 27, 2018

Revised November 23, 2018

Accepted November 27, 2018 